\title{
SAN094.0060 C \\ A COMPUTATIONAL STUDY OF PROJECTILE SHAPE DEPENDENCE ON PHASE CHANGE PHENOMENA WITH COMPARISONS TO EXPERIMENTAL DATA
}

\author{
E. S. Hertel, Jr., ${ }^{1}$ R. L. McIntosh, ${ }^{1}$ and Bruce C. Patterson ${ }^{2}$ \\ 1: Sandia National Laboratories, Albuquerque, New Mexico \\ 2: Wright Laboratory/Armament Directorate, Eglin AFB, FL
}

\begin{abstract}
To make an estimate of the current state of predictive capabilities of hydrocodes for impacts where phase changes may be important, we have simulated a series of experiments where a zinc sphere, rod, and plate impact thin zinc plates at $5 \mathrm{~km} / \mathrm{s}$. The experimental data consists of radiographs of the debris cloud and visual evidence of the damage in an aluminum witness plate. CTH was used to simulate the three experiments noted above. A detailed comparison of the simulated debris structure and subsequent damage will be made with the available data. In general, the CTH predictions match the experimental record.
\end{abstract}

\section{INTRODUCTION}

It has been recognized that the use of shock physics analysis packages (aka, hydrocodes) in the impact regime where materials can be expected to change phase, is strongly dependent on the ability of the hydrocode to accurately model the partitioning of energy and volume change as a result of the pressure induced on impact ${ }^{1}$. The functional form of energy and volume change is commonly referred to as the equation-of-state (EOS) of a material. Other features of the hydrocode, such as the accuracy of the numerical schemes, the ability to model material deformation due to various loading schemes, and failure and fragmentation, are important also. But a key feature is still the decision on how to partition energy and volume change. The EOS of a material can be expressed in terms of an analytic expression or tabular representation of the complex surface. Both methods rely on a combination of theory and experimental data to build the surface representation. The experiments are typically simple flat plate impacts (one-dimensional) that concentrate on complete understanding of a few features. The EOS experiments are useful in understanding the underlying phenomenology of the material, but are generally not useful in validating multi-dimensional features of the hydrocodes. However, it is still important to accurately predict the one-dimensional features typical of EOS experiments to retain a high confidence level for multi-dimensional comparisons. Other experiments that exploit their multi-dimensional characteristics are needed to validate the numerics and physics that are important in conjunction with the EOS experiments.

It is useful to discuss some of the concerns and features surrounding the need for validation experiments in terms of a canonical problem. The canonical problem was chosen so as to illustrate interactions typical of a projectile striking a complex target. From the previous discussion, it should be clear that code validation requires relatively simple but carefully chosen experiments that reflect the key physical processes of the actual but complex interactions.

Figure 1 shows a sketch of the canonical problem for code validation over a range of impact velocities along with diagnostic equipment appropriate for such a problem. This sketch shows a projectile (shown here as a sphere, but any simple shape could be used) impacting a plate. The initial impact will produce a debris cloud whose state will depend on the impact velocity and material characteristics. The debris cloud will then traverse a void space and another impact will occur with a second plate. The secondary impact will also produce a debris cloud whose state will now depend on the characteristics of the initial debris cloud, the secondary impact velocity, and the material characteristics. It should be expected that the secondary debris cloud will have different characteristics than the first cloud. The secondary debris cloud will also traverse a void space and impact a third plate.

A key aspect of impact induced fragmentation is that as the velocity increases, the size distribution of the resulting debris cloud changes. At relatively low impact velocities, the size distribution is skewed towards a few large particles (large being $>10 \%$ of initial projectile mass) with few or no small particles. As the velocity increases, the number of large particles decreases and the number of small (small being $>1 \%$ of initial projectile mass) to very small particles increases. The size of the largest particle also decreases as the total number of particles increases. This progression has interesting ramifications for our canonical problem as is illustrated by the following.

At low impact velocities, a few large fragments are created by the initial impact. This scenario can be visualized in Figure 2. Here large fragments refer to objects much larger (fully resolved) than the computational mesh. The dynamics of the initial impact are dominated 


\section{DISCLAIMER}

This report was prepared as an account of work sponsored by an agency of the United States Government. Neither the United States Government nor any agency thereof, nor any of their employees, make any warranty, express or implied, or assumes any legal liability or responsibility for the accuracy, completeness, or usefulness of any information, apparatus, product, or process disclosed, or represents that its use would not infringe privately owned rights. Reference herein to any specific commercial product, process, or service by trade name, trademark, manufacturer, or otherwise does not necessarily constitute or imply its endorsement, recommendation, or favoring by the United States Government or any agency thereof. The views and opinions of authors expressed herein do not necessarily state or reflect those of the United States Government or any agency thereof. 


\section{DISCLAIMER}

Portions of this document may be illegible in electronic image products. Images are produced from the best available original document. 


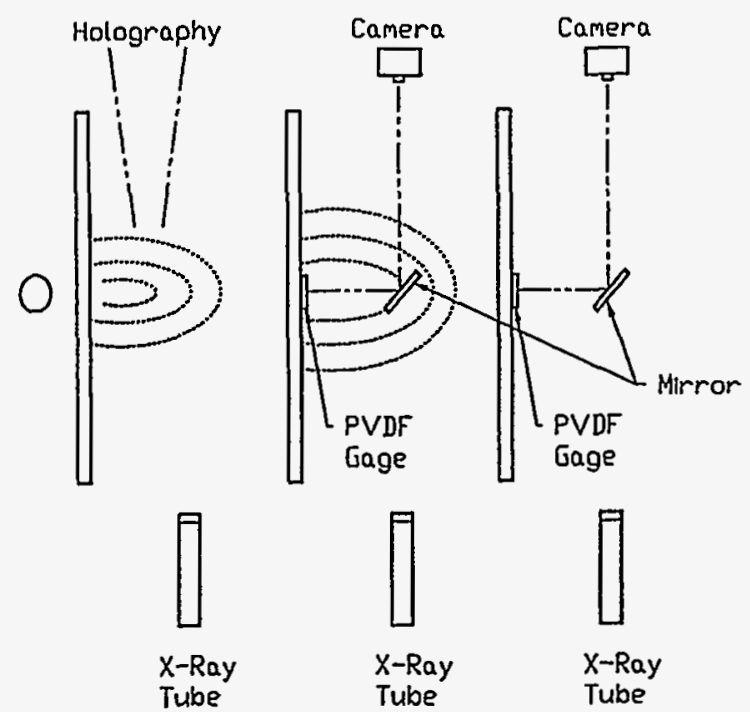

Fig. 1. Canonical Problem Description

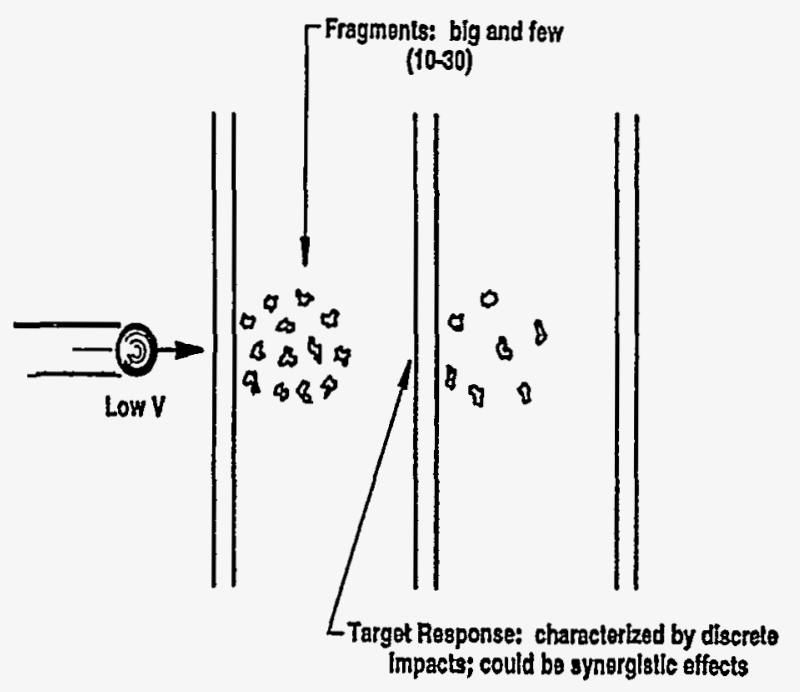

Fig. 2. Conceptual Low Velocity Impact Debris Cloud

by tensile failure phenomena. The secondary impacts are characterized by discrete impacts with little or no synergism and damage at the second plate is dominated by strength. A smaller number of spall generated fragments my be ejected from the back of the second plate. The tertiary impacts are very few and damage at the third plate is again dominated by strength rather than hydrodynamics. As the velocity increases, the initial debris cloud is characterized by a greater number of smaller fragments. This scenario can be visualized in Figure 3. Here the fragments are not larger than the computational mesh, but comparable in size (marginally resolved). The failure regime is still dominated by ten-

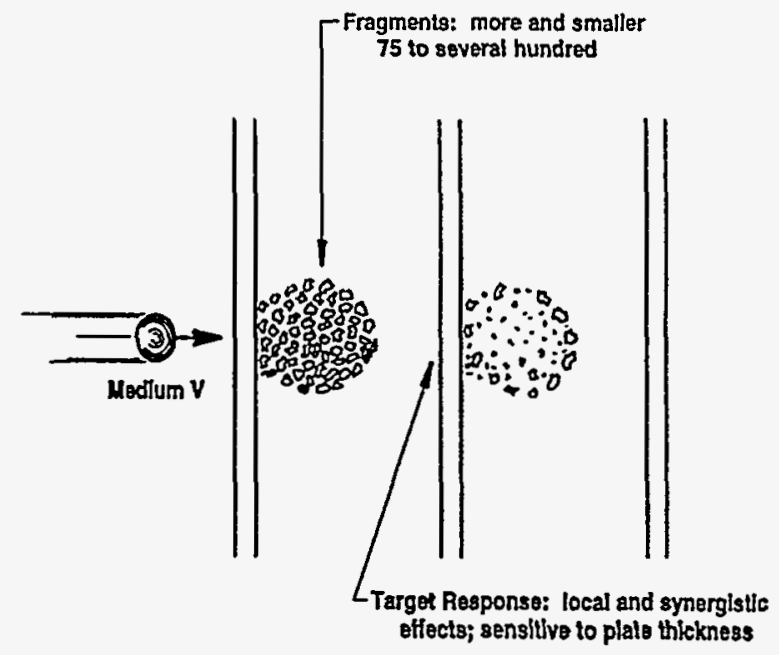

Fig. 3. Conceptual Intermediate Velocity Impact Debris Cloud

sile phenomena, but the process is statistically determined and only averages of size and trajectory have meaning. Depending on material characteristics, the initial debris cloud may contain some melted material along with discrete particles. The secondary target response is determined by a combination of local strength and synergistic effects. The secondary debris cloud may consist of fewer and larger spall induced fragments due to the discrete impacts mixed with particles that survived perforation, or a spall plug due to impulsive loading. Loading on the third plate now resembles secondary loading for a lower velocity initial impact. As the velocity increases again, the initial debris cloud is characterized by many very small fragments. This scenario can be visualized in Figure 4. Here the fragments are much smaller than the computational mesh and are being treated as a continuum of material by the hydrocodes. The failure regime is dominated by tensile phenomena, but the process is statistically determined and only distribution and shape of the debris cloud have meaning. Depending on material characteristics, the initial debris cloud will contain some melted material, some vapor, and some solid particles. The secondary target response is principally determined by impulsive loading. The secondary debris cloud will consist of more and smaller fragments and should be similar to the initial debris cloud of a medium velocity impact. Loading on the third plate now resembles secondary loading for a medium velocity initial impact.

It should be clear from the above discussion that even for high velocity initial impacts, a critical link to lower velocity phenomena is maintained for complex targets. This fact makes it clear that a relatively simple 


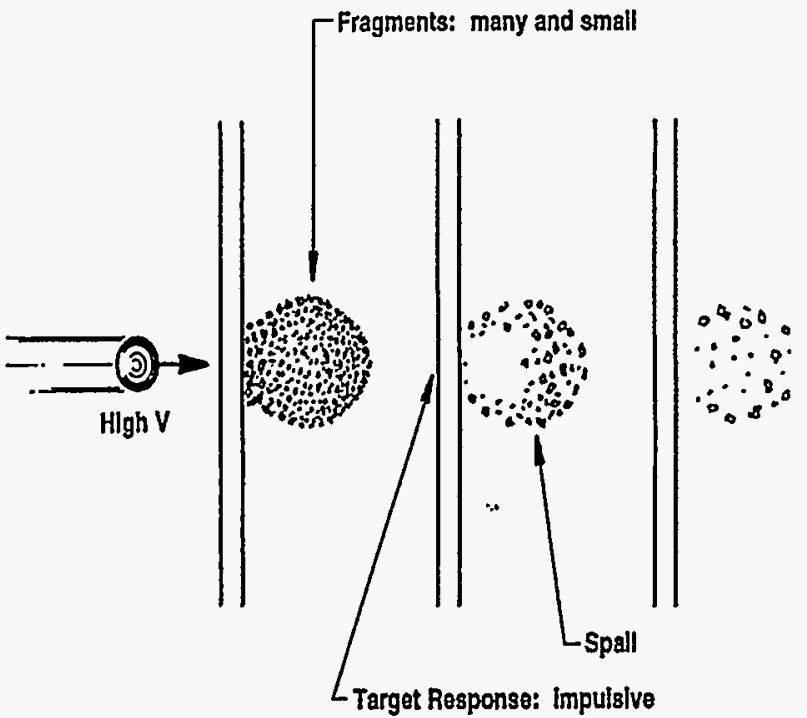

Fig. 4. Conceptual High Velocity Impact Debris Cloud experimental series can be used to simulate many of the important features in complex higher velocity impacts. The shape variation study described here does not meet all of the needs as noted in the discussion above, but given program constraints, we were forced to use currently available experimental data. The comparisons contained in this paper form a valuable addition to the validation of hydrocodes like $C T H$. The ideas contained in this discussion are described in more detail in a joint paper with Anderson ${ }^{2}$.

\section{EXPERIMENTAL SERIES}

An existing series of impact experiments for zinc projectiles impacting a thin zinc plate was described by $\mathrm{Konrad}^{3}$. This series spans a limited impact velocity range of 4.97 to $5.01 \mathrm{~km} / \mathrm{s}$ and varies the projectile type that impacts the witness plate. At this velocity, the debris clouds are composed of very small molten fragments. The debris should not contain vapor as an impact velocity of $\sim 7 \mathrm{~km} / \mathrm{s}$ is required to cause vapor production in zinc. This series of experimental data closely matches the needs of code validation for complex impacts as described in the previous section and will provide the basis for a useful evaluation of hydrocode capabilities as a function of projectile shape when a phase change occurs. The experiments consisted of measurements of damage to the witness plate and debris cloud size and shape after the initial impact. The witness plate damage is measured dynamically during the secondary impact and after recovery of the test samples. The specifics of the experiments used in this study are contained in Table 1. Reproductions of the experimental debris structure are contained in Figures 5,6, and 7. The radiographic images reproduced here were taken at $\sim 19 \mu$ s after impact. Experimental witness plate damage and debris characteristics are shown in Table 2.

The data set available for comparison, although not as complete as other studies, does contain very useful data on phase change phenomena and shape effects. The shape of the debris clouds gives insight into the partitioning of energy upon release from the shocked state.

Table 1. Experimental Series Initial Conditions

\begin{tabular}{cccccc}
\hline Test & $\begin{array}{c}\text { Impact Velocity } \\
(\mathrm{km} / \mathrm{s})\end{array}$ & $\begin{array}{c}\text { Projectile } \\
\text { Characteristics } \\
\text { (diameter } \times \text { thickness) }\end{array}$ & $\begin{array}{c}\text { Witness } \\
\text { Thickness } \\
(\mathrm{mm})\end{array}$ & $\begin{array}{c}\text { Bumper } \\
\text { Thickness } \\
(\mathrm{mm})\end{array}$ & $\begin{array}{c}\text { Plate } \\
\text { Separation } \\
(\mathrm{mm})\end{array}$ \\
\hline 1511 & 5.01 & $\begin{array}{c}13.0 \times 0.759 \mathrm{~mm} \\
0.731 \mathrm{gm}\end{array}$ & $\begin{array}{c}\mathrm{Al} 6061-\mathrm{T} 6 \\
2.4384\end{array}$ & 0.9652 & 152.4 \\
Disk & & $5.77 \mathrm{~mm}$ & $\mathrm{Al} 6061-\mathrm{T} 6$ & 0.9652 & 152.4 \\
1515 & 4.98 & $0.719 \mathrm{gm}$ & 2.4384 & & \\
Sphere & & $3.98 \times 14.2 \mathrm{~mm}$ & $\begin{array}{c}\mathrm{Al} 6061-\mathrm{T} 6 \\
2.4384\end{array}$ & 0.9652 & 152.4 \\
1554 & 4.97 & $1.241 \mathrm{gm}$ & 2.4 & \\
Rod & & &
\end{tabular}

Table 2. Experimental Results

\begin{tabular}{cccccc}
\hline Test & $\begin{array}{c}\text { Impact Velocity } \\
(\mathrm{km} / \mathrm{s})\end{array}$ & $\begin{array}{c}\text { Front Velocity } \\
(\mathrm{km} / \mathrm{s})\end{array}$ & $\begin{array}{c}\text { Radial Velocity } \\
(\mathrm{km} / \mathrm{s})\end{array}$ & $\begin{array}{c}\text { Dynamic Hole Size } \\
(\mathrm{mm} \times \mathrm{mm})\end{array}$ & $\begin{array}{c}\text { Recovered Hole Size } \\
(\mathrm{mm} \times \mathrm{mm})\end{array}$ \\
\hline 1511 & 5.01 & 5.22 & 0.92 & $14.4 \times 13.5$ & $14.5 \times 13.5$ \\
1515 & 4.98 & 4.71 & 2.84 & No Hole & No Hole \\
1554 & 4.97 & 5.28 & 1.93 & $38.1 \times 36.3$ & $35.6 \times 35.6$ \\
\hline
\end{tabular}




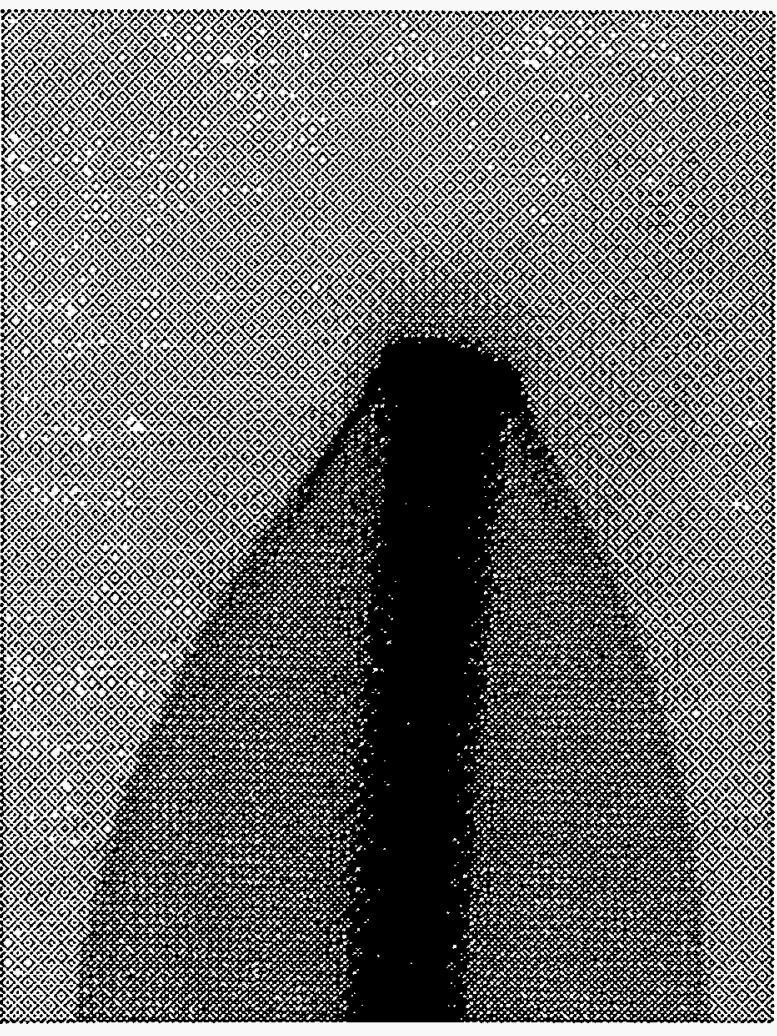

Fig. 5. Experimental Radiograph of the Disk Impact

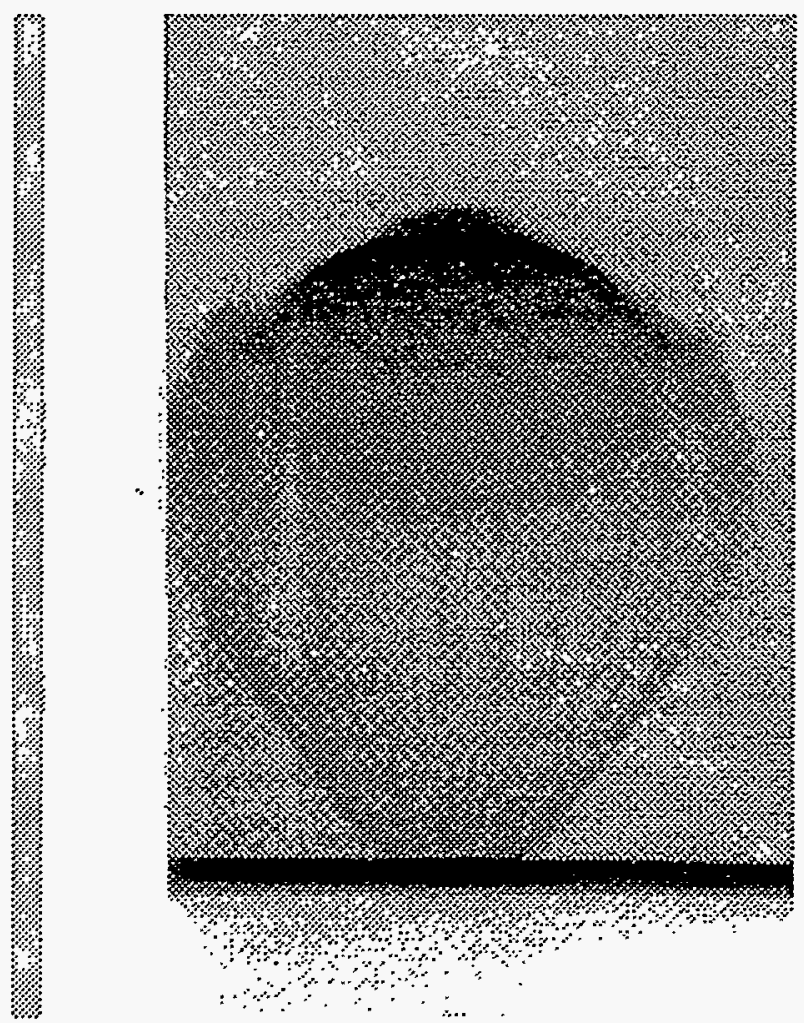

Fig. 6. Experimental Radiograph of the Sphere Impact

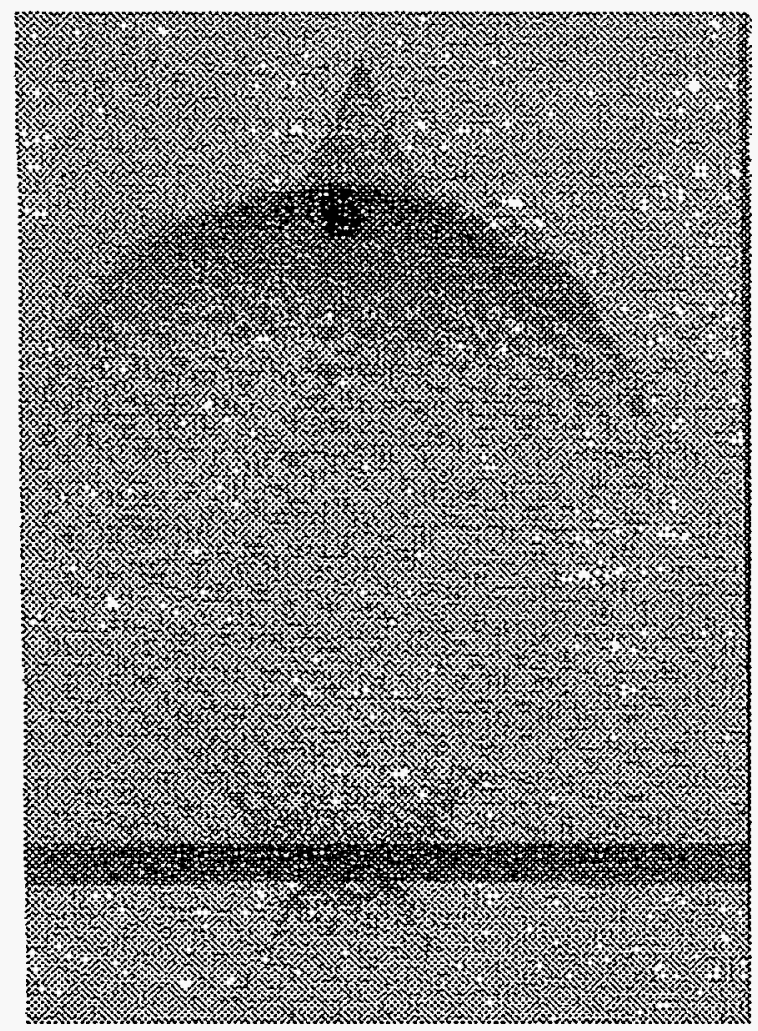

Fig. 7. Experimental Radiograph of the Rod Impact

The witness plate damage characteristics are a sensitive barometer of the transport of debris in condensed material states. Of particular interest is the dynamic versus late-time failure characteristics of the witness plate. A potential difficulty with modelling perforation events is determining when the hole was formed. Late-time failure is very difficult to model with hydrocodes due to the (potentially) very long problem times. Hydrocode estimates of hole size are typically made by assuming all damage occurs at early times and is supported by the experimental evidence in Table 2 for the experiments described here. A careful examination of the experimental and computational record will allow an estimate of the effectiveness of hydrocodes in modelling interactions where phase change, debris transport, and secondary impacts are important as a function of projectile shape.

\section{CTH OVERVIEW}

The $\mathrm{CTH}^{4}$ code was developed to model a wide range of solid dynamics problems involving shock wave propagation and material motion in one, two, or three dimensions: one-dimensional rectilinear, cylindrical, and spherical meshes; two-dimensional rectangular and cylindrical meshes; and three-dimensional rectangular meshes are available. A two-step Eulerian solution scheme is used with these meshes. The first step is a 
Lagrangian step in which the cells distort to follow the material motion. The second step is a remesh step where the distorted cells are mapped back to the original Eulerian mesh. CTH has several thermodynamic models that are used for simulating strong shock, large deformation events. Both tabular and analytic equations-of-state are available. CTH can model material strength, high explosive detonation, fracture, and motion of fragments smaller than a computational cell. Material strength can be modelled as linearly-elastic perfectly-plastic, viscoplastic (strain-rate dependent), or with two different brittle failure models. High explosive detonation can be modelled with a programmed burn model, or two different reactive burn models. A special model is available for moving fragments smaller than a computational cell with the correct statistical velocity. CTH has been carefully designed to minimize the dispersion generally found in Eulerian codes. It has a high-resolution interface tracker that prevents breakup and dispersion generally found in Eulerian codes. In addition, CTH uses a second-order convection scheme to flux all quantities between cells.

\section{CTH RESULTS}

The three experiments described above were the focus of CTH simulations. Each of the three experiments were simulated with $0.01 \times 0.01 \mathrm{~cm}$ (130 to 39 zones across the initial projectile diameters and $\sim 9$ zones through the thickness of the bumper) resolution to study the evolution of the debris cloud before impact with the witness plate; and once with $0.025 \times 0.025 \mathrm{~cm}$ resolution (52 to 16 zones across the initial projectile diameters) to study the entire experiment. The lower resolution used for half of the simulations is still much higher resolution than is considered minimal (4 zones across the smallest dimension) and should give acceptable answers. The low resolution is also similar to other simulations of comparable problems ${ }^{5,6,7}$. Because of computational limitations, the high resolution simulations were completed with a reduced geometric and temporal domain that matched the experimental debris clouds in Figures 5 through 7. The low resolution simulations were used in the prediction of the rear wall damage only. The simulations used an equation-of-state that was developed for a previous study ${ }^{8}$ of zinc vaporization that accurately matched the experimental data. The constitutive model was linearly-elastic, perfectly-plastic with a von Mises yield surface of 1.5 kbars. Failure was initiated by limiting the tensile region of the equationof-state by a $\mathrm{P}_{\min }$ criteria of $3.0 \mathrm{kbars}$. Upon reaching the $\mathrm{P}_{\min }$ criteria, CTH adds void to the computational cell. This relieves the tensile state and allows for the formation of cracks and fragments.

Figures 8,9 , and 10 display the three $\mathrm{CTH}$ simulations (corresponding to Tests 1511, 1515, 1554) of the debris cloud at $20 \mu \mathrm{s}$. The figures display the axi-symmetric simulation reflected about the $\mathrm{x}=0$ axis. The right side of each image displays a simulated radiograph where the density is summed along the line of sight and plotted with a grey scale. The left side of each image displays the material locations where the light grey color represents the original bumper material and the dark grey represents the original projectile material. The simulations were completed with the high resolution computational option described above. Figures 11, 12, and 13 display the three CTH simulations (again corre-

Table 3. Simulated Debris Cloud Comparison with Experimental Data

\begin{tabular}{cccccc}
\hline Test & $\begin{array}{c}\text { Impact Velocity } \\
(\mathrm{km} / \mathrm{s})\end{array}$ & $\begin{array}{c}\text { Experimental } \\
\text { Front Velocity } \\
(\mathrm{km} / \mathrm{s})\end{array}$ & $\begin{array}{c}\text { Computed } \\
\text { Front Velocity } \\
(\mathrm{km} / \mathrm{s})\end{array}$ & $\begin{array}{c}\text { Experimental } \\
\text { Radial Velocity } \\
(\mathrm{km} / \mathrm{s})\end{array}$ & $\begin{array}{c}\text { Computed } \\
\text { Radial Velocity } \\
(\mathrm{km} / \mathrm{s})\end{array}$ \\
\hline 1511 & 5.01 & 5.22 & 5.5 & 0.92 & 1.5 \\
1515 & 4.98 & 4.71 & 5.0 & 2.84 & 3.5 \\
1554 & 4.97 & 5.28 & 5.5 & 1.93 & 3.5 \\
\hline
\end{tabular}

Table 4. Simulated Witness Plate Damage Comparison with Experimental Data

\begin{tabular}{ccccc}
\hline Test & $\begin{array}{c}\text { Impact Velocity } \\
(\mathrm{km} / \mathrm{s})\end{array}$ & $\begin{array}{c}\text { Simulated Hole Size } \\
(\mathrm{mm} \times \mathrm{mm})\end{array}$ & $\begin{array}{c}\text { Dynamic Hole Size } \\
(\mathrm{mm} \times \mathrm{mm})\end{array}$ & $\begin{array}{c}\text { Recovered Hole Size } \\
(\mathrm{mm} \times \mathrm{mm})\end{array}$ \\
\hline 1511 & 5.01 & 15.6 & $14.4 \times 13.5$ & $14.5 \times 13.5$ \\
1515 & 4.98 & No Hole & No Hole & No Hole \\
1554 & 4.97 & 18.8 & $38.1 \times 36.3$ & $35.6 \times 35.6$ \\
\hline
\end{tabular}




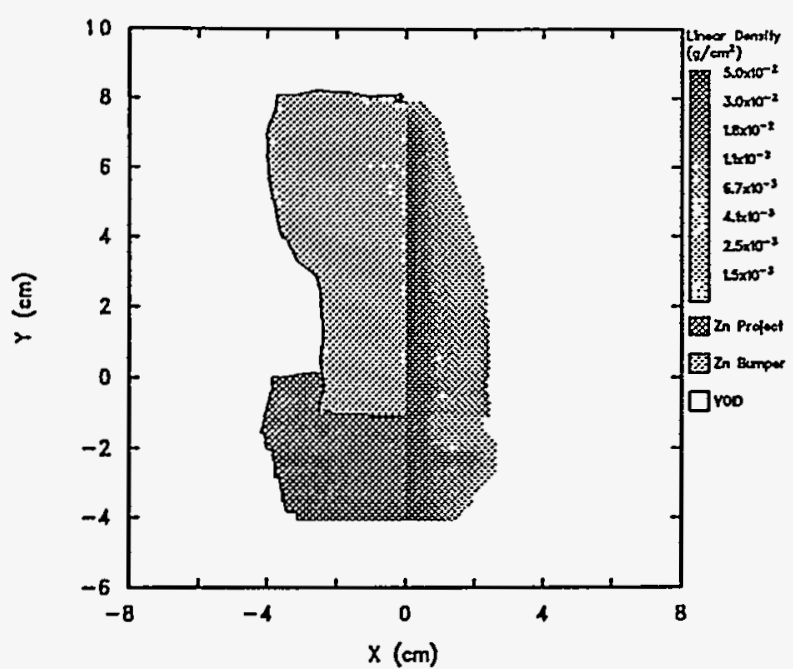

Fig. 8. Simulated Debris Cloud for Test 1511 (Disk)

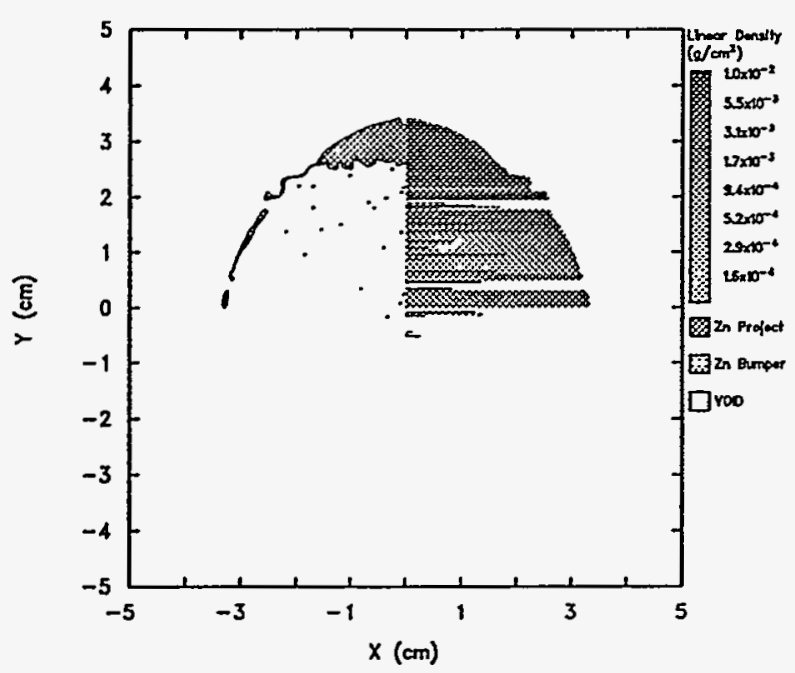

Fig. 9. Simulated Debris Cloud for Test 1515 (Sphere)

sponding to Tests $1511,1515,1554)$ of the debris cloud impacting the witness plate after it has been perforated. These simulations were completed with the low resolution computational option described above. Table 3 contains an estimate of the debris cloud frontal (axial) and radial velocities $20 \mu \mathrm{s}$ after impact and comparisons to the experimental estimates. Table 4 contains the simulated perforation measurements again compared to the experimental results.

\section{DISCUSSION AND CONCLUSIONS}

There are two areas for comparison between the experimental data and the simulated $\mathrm{CTH}$ results: the debris cloud structure (both shape and velocity estimates) and the witness plate terminal danage caused by the initial debris cloud. From the standpoint of the phys-

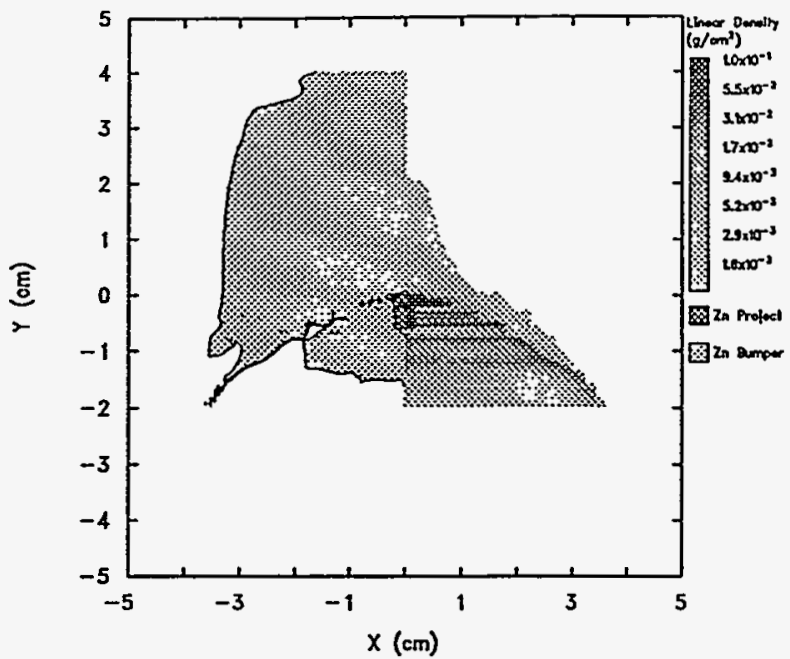

Fig. 10. Simulated Debris Cloud for Test 1554 (Rod)

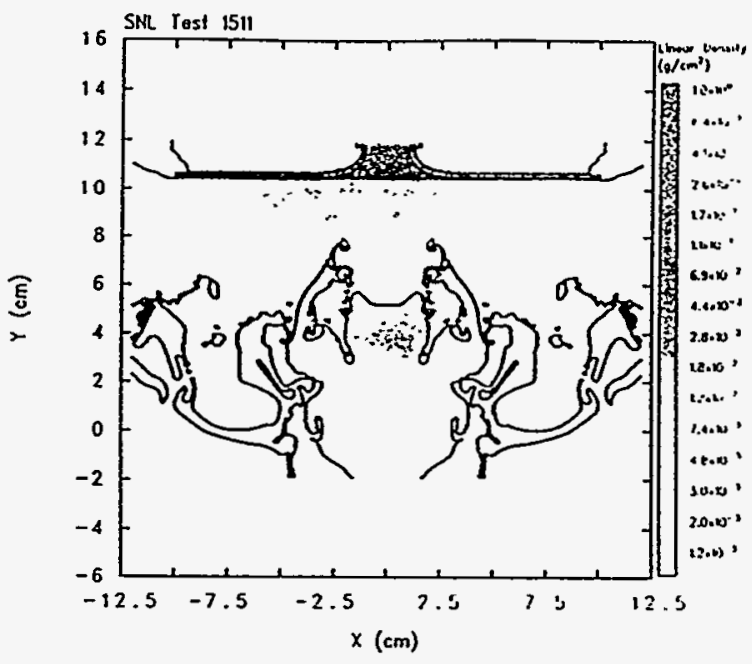

Fig. 11. Simulated Witness Plate Damage for Test 1511 (Disk)

ics of interactions, comparisons of the shape and thermodynamic state of the debris cloud principally test the equation-of-state and constitutive models used. In addition, there are numerical issues surrounding the ability of codes to accurately advect small fragments or vaporous material in the computational mesh that are tested by these types of simulations. The ability to predict secondary damage depends on the debris cloud structure, it's velocity distribution, the coupling of the debris impact into the witness plate, the propagation of the stress waves through the witness plate after impact, and the constitutive and failure models used to represent the witness plate material. One feature discussed in the canonical problem earlier was the generation of a secondary debris cloud. Unfortunately, the experimental 


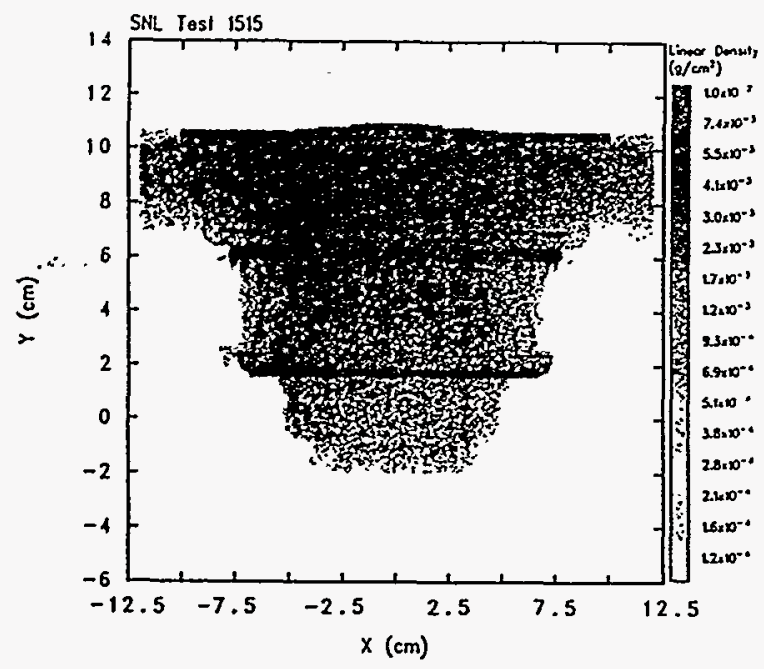

Fig. 12. Simulated Witness Plate Damage for Test 1515 (Sphere)

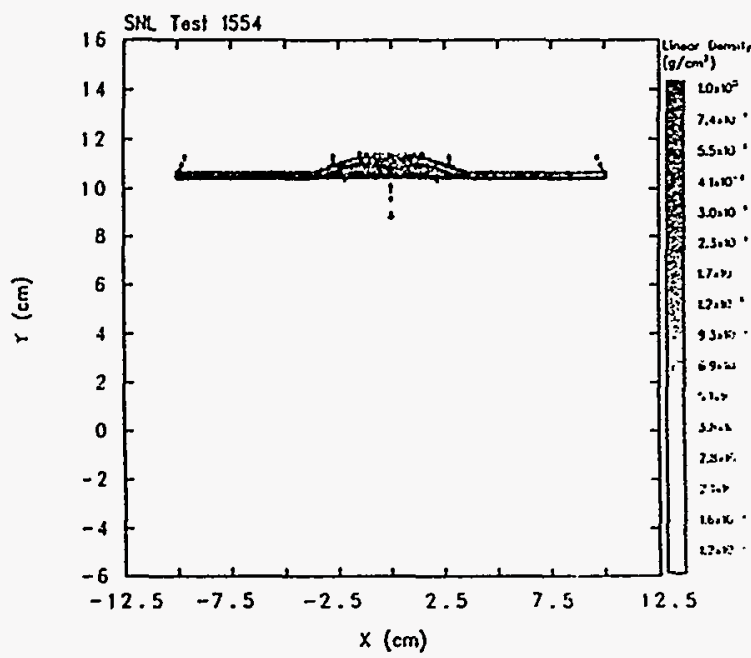

Fig. 13. Simulated Witness Plate Damage for Test 1554 (Rod)

data available does not address this issue directly. This is one area where additional experiments would be useful in determining the accuracy of hydrocodes for this class of problems. However, comparisons with the witness plate damage can be used in an initial understanding of the secondary debris cloud.

The first area for comparison is the debris cloud shape and velocity distribution. The velocity distribution is important since it gives an indication of the accuracy of the time evolution of the cloud. CTH does an excellent job of capturing the key aspects of the experimental record. Figures 5 and 8,6 and 9 , and 7 and 10 can be used to compare the debris shape at approximately $20 \mu \mathrm{s}$. In Figure 5, the distinct interior column of dense material seen experimentally is fully captured in the CTH simulation (Figure 8). In Figure 6, the characteristic (nearly) spherical debris cloud due to a sphere impacting a thin plate is also captured along with the dual radius structure at the front of the cloud (Figure 9). A previous study ${ }^{9}$ by the same authors of spherical projectile impacts into thin plates with varying initial velocities showed similar agreement with the experimental structure. In Figure 7, the bulk of the debris cloud is spherical with a characteristic low density conical front structure. The conical structure is due to the two-dimensional nature of the shock induced into the projectile upon impact. Since the rod is "long" in relation to the shock propagation characteristic, the tail of the rod is left intact. That remnant can seen in the radiograph just behind the spherical front. Again, the CTH simulations (see Figure 10) show a remarkable correspondence with the experimental data.

Table 3 lists the debris cloud velocity estimates for the experiment and the simulations. The CTH simulations capture the key differences between the three shapes investigated here. The simulations and experiments show a relatively constant front velocity that is approximately equal to the impact velocity. CTH overpredicts the front velocity by $\sim 5 \%$. This result would indicate that there is little or no vapor generated and consequently, no phase separation indicated. The radial velocity increases from a very small value for the disk where two-dimensional effects are negligible to approximately half of the impact velocity for the sphere and rod. The simulations match the trends and magnitudes seen in the experiment with the simulations over-predicting the magnitude of the velocity in all cases.

The second area for comparison is in the witness plate damage characteristics. Table 4 shows the comparison between the experimental and simulated witness plate damage. Excellent agreement between the simulated and experimental data exists for the disk and sphere impacts. For Test 1511 (disk impact), the damage is due to the impact of the high density interior column that is well replicated by CTH. The remainder of the debris cloud is very diffuse and causes little damage. For Test 1515 (sphere impact), the debris cloud has diffused due to the significant radial velocity and causes little damage. For Test 1554 (rod impact), the debris cloud has spread radially, although less than the sphere debris cloud. The tail-end of the rod is nearly intact and causes a prompt perforation of the witness plate. The remainder of the debris cloud loads the witness plate near the secondary impact location and causes a pedaling type failure after the initial perforation by the rod remanent. The initial perforation is captured by the CTH simulation but the detailed description of the mechanics necessary to predict a petal type failure is not currently 
included in CTH. Inspection of Figure 13 shows a region of significant deformation (see A) that, if failed, would double the predicted hole size. This would bring the CTH prediction for the rod impact in line with the other two experiments. Further analysis with more sophisticated failure models should be performed to better understand this discrepancy.

In general the agreement between CTH and the experimental data is very good. The only major difference between the CTH simulations and the experimental data is in the hole size for a rod impact. Some of these differences could be due to the simple constitutive (linear-elastic, perfectly-plastic) and failure $\left(\mathrm{P}_{\min }\right.$ with void insertion) models used in the calculations. We feel that the agreement is acceptable given the sophistication of the material models. This study indicates that CTH can accurately predict the structure of a debris cloud for a variety of projectile shapes where melting has occurred. Damage to a witness plate due to secondary impacts can also be predicted accurately, although some discrepancies were noted where complicated dynamical material deformation is important.

Increasing the impact velocity could lead to further changes in predictive capabilities. A previous study ${ }^{9}$ where impact velocities of $\sim 7 \mathrm{~km} / \mathrm{s}$ were studied indicated that CTH can replicate the size and shape of the initial debris cloud accurately for zinc impacting zinc. The form of the debris cloud at this velocity is similar to that shown in Figure 9, although, the mass density of the cloud was lower at the higher impact velocity. The reduced density would indicate that the loading on the secondary target would be strongly time dependent and impulsive. Time dependent impulsive loading places a different requirement on the treatment of the constitutive behavior of the target material. Our belief is that the details of the constitutive behavior may become more important as the velocity increases, especially at or near the "ballistic limit."

\section{REFERENCES}

1. T. G. Trucano Equation of State and Fragmentation Issues in Computational Lethality Analysis, Sandia National Laboratories SAND92-2397 (1993).

2. Anderson, Jr., C. E., E. S. Hertel, Jr., S. A. Mullin, and R. L. Hunt, The Hypervelocity Experimental Research Database (HERD): Support for the Defense Nuclear Agency Code Validation Program, to appear in the 1994 AIAA Space Programs and Technologies Conference (1994).

3. C. H. Konrad, L. C. Chhabildas, M. B. Boslough, A. J. Piekutowski, K. L. Poormon, S. A. Mullin, and D. L. Littlefield, "Dependence of Debris Cloud Formation on Projectile Shape," Proceedings of the 1993 Topical Conference on Shock Waves in Condensed Matter, in press (1993).

4. Hertel, Jr., E. S., R. L. Bell, M. G. Elrick, A. V. Farnsworth, G. I. Kerley, J. M. McGlaun, S. V. Petney, S. A. Silling, P. A. Taylor, and L. Yarrington CTH: A Software Family for Multi-Dimensional Shock Physics Analysis, Proceedings of the 1993 International Symposium on Shock Waves (1994).

5. Chhabildas, L. C. and E. S. Hertel, Jr. Comparison of Analytic Whipple Bumper Shield Ballistic Limits with CTH Simulations, Sandia National Laboratories SAND92-0347 (1992).

6. Hertel, Jr., E. S., L. C. Chhabildas, and S. A. Hill, Whipple Bumper Shield Simulations, in Shock Waves in Condensed Matter-1991, edited by S. C. Schmidt, J. W. Jorbes, and R. D. Dick, Elsevier Science Publishers B. V. (1992).

7. Hertel, Jr., E. S., Simulations of Orbital Debris Impacts on Bumper Shields, Proceedings of the First European Conference on Space Debris (1993).

8. Wise, J. L., G. I. Kerley, and T. G. Trucano, ShockVaporization Studies on Zinc and Porous Carbon, in Shock Waves in Condensed Matter-1991, edited by S. C. Schmidt, J. W. Jorbes, and R. D. Dick, Elsevier Science Publishers B. V. (1992).

9. E. S. Hertel, Jr., R. L. McIntosh, and B. C. Patterson, "A Comparison of Phase Change Phenomena in CTH with Experimental Data" Proceedings of the 1994 Hypervelocity Impact Symposium, accepted for publication (1994).

This work performed at Sandia National Laboratories supported by the U. S. Department of Energy under contract DE-AC04-94AL85000. Funding for the simulations was provided by the Wright Laboratory at Eglin Air Force Base. 